\title{
Clinical pathology
}

\section{Laboratory testing for poisoning in practice: how, when and why we test for toxins}

\section{Nick Carmichael}

There are in excess of 10,000 inquiries made annually to the Veterinary Poisons Information Service regarding suspected cases of intoxication in the UK and this figure is likely to reflect only a small proportion of the cases which are occurring.

Poisoning in dogs most commonly results from accidental ingestion of toxic material found in the animal's environment. Pet owners, however, often have concerns regarding intentional malicious poisoning. As a result, the need for testing can either be to identify a specific (but often unknown) toxic agent or to help exclude the possibility of poisoning.

The early collection of samples in cases of suspected intoxication is critical, as excretion and clearance of any toxin or its metabolites may be well advanced by the time clinical signs emerge. Consequently, routine collection and storage of samples taken on admission from all possible intoxication cases is recommended. If other possibilities are excluded during the case work-up these initial samples may represent the only material from which toxins can be identified.

The preferred samples comprise the suspected toxic material itself or vomitus (owners should be advised to bring these to the practice if available), gastric washing fluid, heparin whole blood $(5 \mathrm{ml})$ and plain urine $(5 \mathrm{ml})$. In cases of historic or chronic toxin ingestion hair analysis can also be useful.

Animals which have died can still provide diagnostic samples from cardiocentesis and cystocentesis and, if postmortem is undertaken, fresh liver, kidney and abdominal fat should be frozen and retained independent of fixed tissues for histopathology. Fixed tissues and faecal material are not generally suitable for toxicological analysis.

The samples should be clearly labelled, dated and stored frozen. If there is any possibility of legal proceedings it is essential that a written chain-of-evidence form is completed, witnessed and submitted along with the samples. The samples and paperwork for these cases must be sent either by an individually tracked courier service or Royal Mail Special Delivery to maintain the chain of evidence from the practice to the laboratory.

Within the laboratory chromatographic methods using gas or liquid are initially used to separate the molecules and compounds in the samples. A range of detection systems including mass spectrometry (MS) or UV absorption are used to further analyse the separated molecules. In MS these individual components are ionized and the resulting spectrum of ions is recorded. Combining this data with the time of emergence from the chromatography and comparing it against known databases allows up to 50,000 individual compounds to be screened for in one analysis.

\section{KEY LEARNING OBJECTIVES}

- Appreciate the critical importance of early sample collection in potential cases of poisoning

- Understand the requirements for the appropriate collection, storage, labelling, chain-of-evidence compliance and submission of samples for analysis from suspected poisoning cases

- Outline the major analytical methods used in toxicology and how these relate to the likelihood of detection of toxins from suspected cases

\section{MULTIPLE CHOICE QUESTIONS}

1. Which of the following would be unlikely to be associated with signs of intoxication following ingestion in dogs?
(A) Dark chocolate
(B) Bunch of grapes
(C) Metaldehyde
(D) PVA glue

2. Which of the following statements about gas chomatography-mass spectrometry (GC-MS) analysis is UNTRUE?

(A) GC-MS analysis has the ability to identify thousands of compounds in a single sample

(B) GC-MS analysis can identify the toxic agents associated with intoxication with lilies in samples from affected cats

(C) GC-MS can identify some but not all organophosphates

(D) GC-MS can identify cannabis in cases of intoxication in dogs

3. Which of the following samples would be most likely to be diagnostic for exposure to drugs of abuse ingested 24 hours previously?
(A) Urine
(B) Gastric contents
(C) Faeces
(D) Heparin whole blood 